\title{
Mass-array screening of frequent mutations in cancers reveals $R B 1$ alterations in aggressive adrenocortical carcinomas
}

\author{
Bruno Ragazzon 1,2,3, Rossella Libé 1,2,3,4,5, Guillaume Assié 1,2,3,4, \\ Frédérique Tissier ${ }^{1,2,3,6,11}$, Olivia Barreau 1,2,3,4, Claude Houdayer ${ }^{3,7,8}$, \\ Karine Perlemoine 1,2,3, Anne Audebourg6 ${ }^{6}$, Eric Clauser9 , Fernande René-Corail 1,2,3, \\ Xavier Bertagna ${ }^{1,2,3,4}$, Bertrand Dousset ${ }^{10}$, Jérôme Bertherat ${ }^{1,2,3,4}$ and \\ Lionel Groussin 1,2,3,4
}

${ }^{1}$ INSERM, U1016, Institut Cochin, Paris, France, ${ }^{2}$ CNRS, UMR8104, Paris, France, ${ }^{3}$ Université Paris Descartes, Sorbonne Paris Cité; 24, Rue du Faubourg Saint Jacques, 75014 Paris, France, ${ }^{4}$ Department of Endocrinology, Referal Center for Rare Adrenal Diseases, Assistance Publique Hôpitaux de Paris, Hôpital Cochin, 27, Rue du Faubourg Saint Jacques, 75014 Paris, France, ${ }^{5}$ French COMETE Network for Adrenal Cancer, Paris, France, ${ }^{6}$ Department of Pathology, Assistance Publique Hôpitaux de Paris, Hôpital Cochin, Paris, France, ${ }^{7}$ Service de Génétique Oncologique, Institut Curie, Paris, France, ${ }^{8}$ Inserm, U830, Centre de Recherche de I'Institut Curie, Paris, France, ${ }^{9}$ Oncogenetic Laboratory, ${ }^{10}$ Department of Digestive and Endocrine Surgery, Assistance Publique Hôpitaux de Paris, Hôpital Cochin, Paris, France and ${ }^{11}$ Department of Pathology, Groupe Hospitalier Pitié-Salpétriére, Assistance Publique Hôpitaux de Paris, Université Pierre et Marie Curie (Paris VI), Paris, France

Correspondence
should be addressed
to L Groussin
Email
lionel.groussin@cch.aphp.fr

\begin{abstract}
Context: Adrenocortical carcinoma (ACC) is a rare disease with a poor overall outcome. Transcriptome analysis identified two groups of ACCs with different prognosis. In aggressive ACCs, somatic mutations of the tumor suppressor gene TP53 and the proto-oncogene $\beta$-catenin are detected in $50 \%$ of cases. For the remaining aggressive ACCs and for the group with a better prognosis, molecular alterations are unknown.

Objective: To identify new molecular actors driving adrenal tumorigenesis.

Experimental design: Analysis by mass array of 374 mutations among 32 common oncogenes or tumor suppressor genes was performed on the tumoral DNA of 26 ACCs, using Sequenom OncoCarta Panels.

Results: Four mutations were identified, two previously known $\beta$-catenin mutations and one alteration in two other genes: $J A K 3$ and retinoblastoma gene (RB1). The JAK3 alteration was found in leukocyte DNA and therefore considered as a polymorphism and not a somatic event. The full $R B 1$ tumor suppressor gene was subsequently sequenced in a cohort of 49 ACCs (26 ACCs from the 'OncoCarta cohort' and 23 other ACCs): three somatic mutations were identified, all in the poor-outcome ACC group. By immunohistochemistry, a loss of the retinoblastoma protein ( $p R b)$ was found exclusively in aggressive ACCs in $27 \%$ of cases (seven out of 26 ), three of them with an inactivating RB1 mutation. Among the seven pRb-negative ACCs, five had an allele loss at the RB1 locus.

Conclusions: Parallel analysis of somatic mutations among known cancer genes allowed us to identify $R B 1$ as a new actor in aggressive ACCs. These results suggest a prognostic significance of pRb expression loss in ACCs.
\end{abstract}

\section{Introduction}

Adrenocortical carcinoma (ACC) is a very rare neoplasia arising from the adrenal cortex with an incidence of one to two cases per million per year. The overall prognosis is poor with an overall survival rate of $38 \%$ at 5 years (1). Recently, transcriptome analysis carried out independently by two different teams, including ours, revealed
(ㄷ) 2014 European Society of Endocrinology Printed in Great Britain
Published by Bioscientifica Ltd. 
that two subgroups of ACCs with a completely opposite prognosis could be distinguished $(2,3)$. Adrenocortical tumorigenesis is partially understood. Somatic alterations of three key players in carcinogenesis have been well characterized in ACCs: a frequent overexpression of insulin-like growth factor 2 (4), inactivating mutations of the tumor suppressor gene TP53 (5), and activating mutations of the proto-oncogene $\beta$-catenin (CTNNB1) $(6,7)$. The poor-prognosis ACC group is partially explained by the TP53 and CTNNB1 mutations, which are only found in aggressive ACCs with a mutually exclusive pattern (8).

To help define the prognosis and find medication for patients with a metastatic disease, there is an urgent need to identify new pathways driving adrenocortical carcinogenesis. For this purpose, we decided to perform a somatic mutation profiling on tumoral DNA from ACCs. We used a sensitive method based on massive parallel sequencing of a large panel of well-known genes involved in carcinogenesis (Sequenom's OncoCarta). This method enables sensitive and rapid somatic mutation profiling in solid tumors or cell lines. Previous studies have validated the accuracy of Sequenom's OncoCarta to identify mutations in most of the oncogenes analyzed, with the expected frequency $(9,10,11)$. In this work, this technique allowed us to reveal the disruption of the key retinoblastoma protein $(\mathrm{pRb})$ pathway in adrenocortical carcinogenesis.

\section{Subjects and methods}

\section{Tumor samples}

Forty-nine tumors were prospectively collected, and DNA was extracted as previously described (2). The study was approved by the Institutional Review Board of Cochin Hospital. Each patient was fully informed and signed a written agreement. In a previous work, unsupervised analysis of transcriptomic profiles revealed two groups of ACCs with two opposite outcomes $(2,3)$ : 41 differentially expressed genes were studied by quantitative RT-PCR. A prognosis predictor was built based on the combination of four genes: MCM5, VEPH1, PINK1, and SLC2A1. The predictor was a powerful tool to classify an extended cohort of ACCs in the poor or better prognosis groups (8). Characteristics of the ACC cohort are shown in Fig. 1.

\section{OncoCarta assay}

Among the 49 ACCs, 26 ACC tumors were analyzed by Sequenom's OncoCarta Panel v1.0 and v3.0 (Sequenom, Hamburg, Germany). These panels provide a useful tool for sensitive mutation screening by parallel analysis of 374 simple and complex mutations among 32 common oncogenes and tumor suppressor genes (see www. sequenom.com).

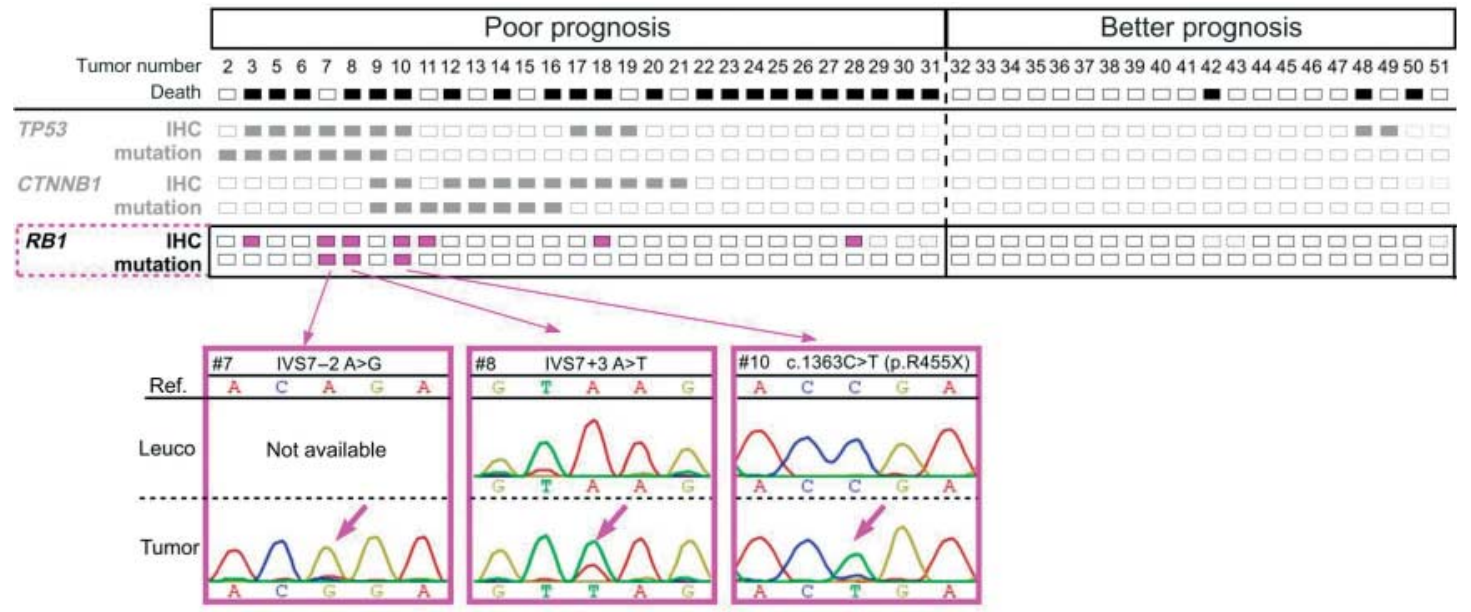

\section{Figure 1}

RB1 alterations in the whole cohort of 49 ACCs. The ACC cohort was divided into two groups according to the clinical outcome (29 poor-outcome ACCs and 20 ACCs with a better prognosis). The clinical status of patients at the last follow-up is indicated by a rectangle (black for dead and white for alive). The seven ACCs with a negative $\mathrm{pRb}$ immunostaining (IHC) and the three
RB1 mutated ACCs are denoted as pink rectangles. DNA sequence chromatograms of leukocyte (Leuko) and tumor are shown with the reference sequence (ref.). The previously identified TP53 and CTNNB1 alterations are denoted as gray rectangles (8). 


\section{Sanger sequencing}

The $R B 1$ promoter and the 27 exons were sequenced in the entire cohort of 49 ACCs, using primers previously described (12). Sequencing was performed twice for each sample harboring a potential mutation.

\section{Immunohistochemistry studies}

Immunohistochemical staining for $\mathrm{pRB}$ was done using an anti-RB1 antibody (NCL-RB clone 1F8, Novocastra, Newcastle, UK). Paraffin-embedded tissue sections were available for 43 out of 49 tumors. Manufacturer's instructions were followed. A loss of pRB expression was considered only when a negative staining of the adrenocortical cells was associated with a positive staining of endothelial cells (positive control of the antibody). The immunohistochemistry analysis was carried out with the same technique described by Sharma et al. (13). A loss of $R B 1$ expression was considered only if nuclear $\mathrm{pRB}$ immunodetection scored negative.

\section{RB1 promoter methylation and loss of heterozygosity analyses}

Global $R B 1$ promoter methylation of $\mathrm{CpG}$ islands was analyzed using a PCR-based methylation assay. Tumoral DNA was digested with the methylation-sensitive enzyme CfoI, which is inhibited by cytosine methylation of its recognition site (GCGC). RB1 promoter with CpG islands and a control fragment from the $A F M$ gene without $\mathrm{CpG}$ were co-amplified by PCR with the AmpliTaq Gold (Applied Biosystems). The following primers were used for the $R B 1$ promoter: CTGGACCCACGCCAGGTTTC and GTTTTGGGCGGCATGACGCCTT (343 bp) and AFM: AGCTTTTGAAAGCAGAGTCA and GTAACGGTAACAACCTGGAG (238 bp). $R B 1$ promoter amplification occurs only when CpG sites are methylated and therefore uncut by CfoI. CpGenome Universal Methylated DNA (S7821, Chemicon, Millipore, Billerica, MA, USA) was used as a methylation-positive control. PCR products were analyzed by agarose gel electrophoresis.

Allelic loss at the RB1 locus was evaluated using three microsatellite markers (D13S153, RBi4, and D13S284). The three microsatellites were chosen because of their high informativity and location close to and within $R B 1$ gene. Briefly, leukocyte and tumor DNA were amplified by PCR with the use of fluorescent-labeled primers. The analysis of the PCR product was done with an automatic sequencer (model CEQ 8800, Genetics Analysis System version 8.0; Beckman Coulter, Fullerton, CA, USA).

\section{Expression analysis}

The expression profiles derived from a previous study (2) were performed with the HG-U133 Plus 2.0 Affymetrix GeneChip arrays (ArrayExpress web site: http://www.ebi. ac.uk/arrayexpress, experiment E-TABM-311). In order to investigate the consequences of $\mathrm{pRb}$ loss on the expression of E2F-pRB target genes, we analyzed the transcriptome data of 29 ACCs in which pRb immunohistochemistry was carried out. Analyses were carried out using ' $R$ ' and the Limma test was used for group comparisons (LIMMA $\mathrm{R}$ package) as previously described (8).

\section{Results}

\section{Mutation profiling of known cancer genes (OncoCarta cohort)}

A parallel analysis of 374 somatic mutations among 32 common oncogenes and tumor suppressor genes was carried out on 26 ACCs tumor DNA with two different OncoCarta panels (v1.0; v3.0). Four mutations were identified. Two CTNNB1 mutations ( $\beta$-catenin mutation pS45F) were known from a previous study (6). Interestingly, two other genes were found with a genetic alteration: the JAK3 gene (nonsynonymous alteration p.V722I, ACC no. 8) and the retinoblastoma gene (RB1) (c.1363C > T, nonsense mutation p.R455X, ACC no. 10).

By direct Sanger sequencing, the JAK3 and RB1 genetic alterations were confirmed in tumor DNA. In order to determine if these alterations were a somatic event, the corresponding leukocyte DNA was sequenced. The $J A K 3$ alteration was found in the leukocyte DNA. Moreover, in the database of single nucleotide polymorphisms (dbSNP, rs3213409) and in the Catalog Of Somatic Mutations In Cancer (COSMIC, COSM34213), this genetic event is considered as a possible polymorphism. For this reason, we decided to focus our attention on the retinoblastoma gene.

\section{Direct $R B 1$ sequencing in a large series of 49 ACCs}

As only ten different $R B 1$ mutations were screened by the OncoCarta panels, the entire $R B 1$ gene was sequenced on tumor DNA in the 26 ACCs (OncoCarta cohort) and in 23 additional ACCs. A total of six RB1 genetic alterations were found in five different ACCs; two of them (IVS12-29 $A>G$ and c.1574 $C>G, p A 525 G$ ) were identified in the same carcinoma (ACC no. 16). These two genetic variations are neutral variants (dbSNP rs3092886 and 


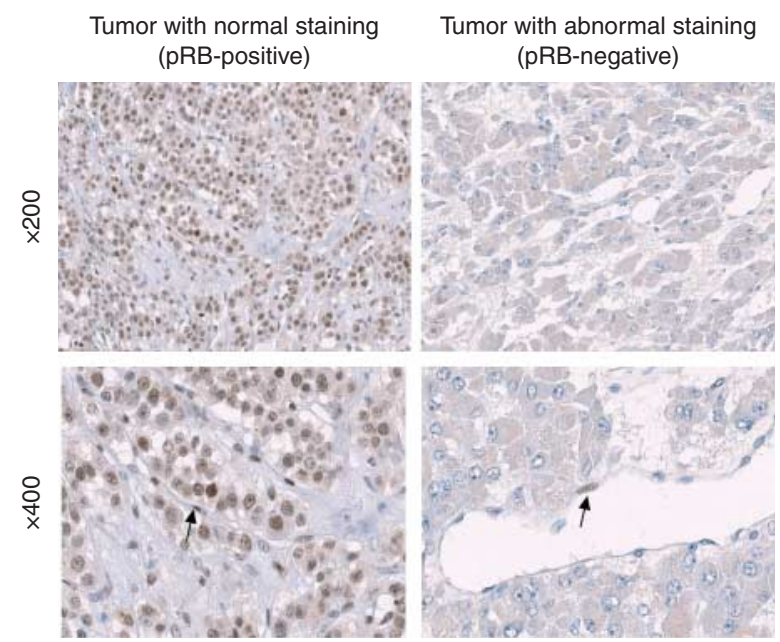

Figure 2

pRB immunohistostaining in ACCs. Immunohistochemistry in a good prognosis ACC showing a normal pRb nuclear staining (left), in comparison with an aggressive ACC (no. 11) showing negative pRb expression (right). Arrow showed stromal cells used as positive-staining control. Magnification, $\times 200$ and $\times 400$.

rs4151539 respectively) and were therefore identified in the corresponding leukocyte DNA. A third genetic variation, an undescribed base substitution in intron 3 (IVS3 + 46 G>A, ACC no. 11), was found in the patient leukocyte DNA. In silico splice predictions (analyzed by using six tools as previously described $(14,15)$ ) were negative, therefore this variation was not considered as a somatic mutation.

Among the three remaining genetic abnormalities, two were detected in splicing consensus sites (IVS7-2 A $>$ G, ACC no. 7 and IVS7 + 3 A $>$ T, ACC no. 8; Fig. 1) and were predicted to be highly damaging. The IVS7 $-2 \mathrm{~A}>\mathrm{G}$ mutation was previously identified in a family with retinoblastoma (16) but the IVS7 $+3 \mathrm{~A}>\mathrm{T}$ is not reported in the COSMIC database. The nonsense mutation (p.R455X, ACC no. 10) was previously reported in several tumor types (COSM895). Finally, three out of 49
ACCs $(6 \%)$ were considered to carry an inactivating $R B 1$ somatic mutation.

\section{Immunohistochemistry, promoter methylation, and allelic loss studies}

To determine if the pRb expression was altered in ACC, immunohistochemical staining was studied on 43 available tissue samples among the 49 ACCs. pRb nuclear staining was totally lost in the tumoral cells from seven ACCs (16\%), three of which harboring a $R B 1$ inactivating mutation (Figs 1 and 2).

In order to explain the $\mathrm{pRb}$ lost in the seven tumors we carried out promoter methylation and loss of heterozygosity ( $\mathrm{LOH}$ ) analyses. No promoter hypermethylation was observed for these seven tumors (Fig. 3). For ACC no. 10 , the allele frequency of the mutated allele $(81 \%$, c. $1363 \mathrm{C}>\mathrm{T}$, p.R455X) in the mass-array screen was in favor of a WT allele loss in the tumor. The $R B 1$ sequence electropherogram of the tumor DNA for the three mutated ACCs was also in favor of a complete (ACC no. 7 and ACC no. 10) or relative (ACC no. 8) loss of the WT RB1 allele in the tumor (Fig. 1). Moreover, by analyzing the informative microsatellite markers in the $\mathrm{LOH}$ study, we observed that two more ACCs (ACC no. 3 and ACC no. 18) among the seven with a pRb loss had an allelic loss (Fig. 4).

\section{All RB1 alterations were found in the poor-outcome ACC group}

By integrating the previous data with the subclassification of the whole ACC cohort in two groups (29 ACCs with a poor and 20 ACCs with a better prognosis) (8), we observed that the $R B 1$ genetic and $\mathrm{pRB}$ expression alterations were only associated with the poor-outcome ACC group (Fig. 1). As a consequence, $10 \%(3 / 29)$ of the poor-outcome ACCs were carriers of a somatic $R B 1$ mutation. In a similar way, $27 \%(7 / 26)$ of the poor-prognosis ACCs were found with a pRb loss.

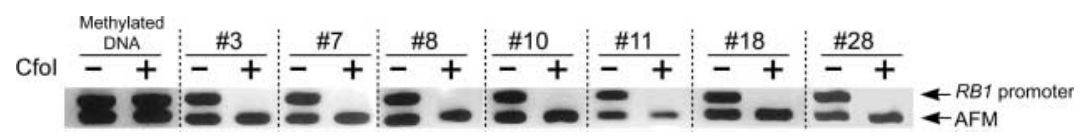

\section{Figure 3}

Assay for methylation status of the RB1 promoter. DNA from the seven tumors with $\mathrm{pRb}$-negative staining was predigested with Cfol enzyme $(+)$ or without (-). RB1 promoter and control (AFM) were co-amplified with specific primers and separated by agarose gel electrophoresis. A methylated DNA was used as a positive control. None of the pRb-negative tumor had promoter hypermethylation. 

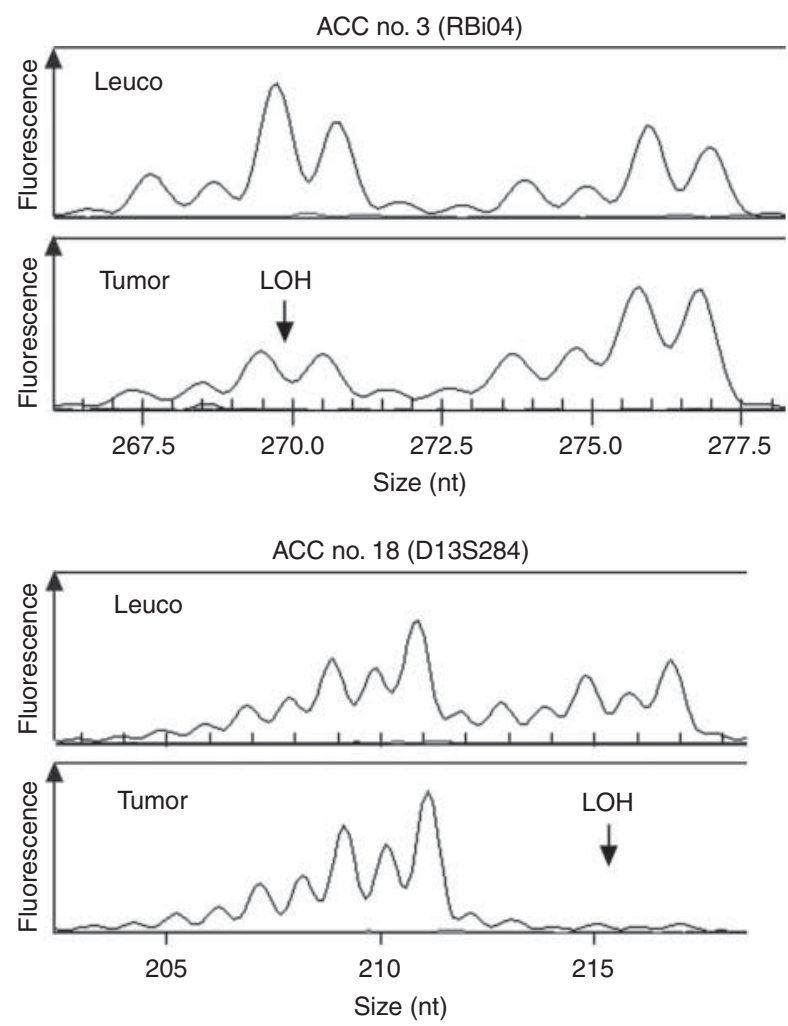

Figure 4

RB1 loss of heterozygosity ( $\mathrm{LOH}$ ) analysis. Capillary electrophoresis pattern of the polymorphic microsatellites markers Rbi04 and D13S284 from leukocyte and tumoral DNA of ACC no. 3 and ACC no. 18. LOH was detected in tumoral DNA in comparison with leukocyte DNA. Arrows show the allele loss in the two ACCs.

Expression of E2F-pRB target genes (Supplementary Table 1 , see section on supplementary data given at the end of this article) was altered in the seven ACC with pRB loss. An overexpression of cell-cycle effectors negatively regulated by pRb, such as CHEK1 (checkpoint kinase 1), CCNE2 (cyclin E2), and MCM6 (minichromosome maintenance complex) was observed $(P<0.001 ;$ Fig. 5).

Furthermore, and by contrast with TP53 and CTNNB1 abnormalities, RB1 alterations were not found with a mutually exclusive pattern (Fig. 1 and Supplementary Table 2 , see section on supplementary data given at the end of this article). It is worth noting that four ACCs had a TP53 and a RB1 alteration simultaneously.

\section{Discussion}

Somatic mutation screening among known cancer genes allows to quickly identify genetic markers in cancer pathways. Using a similar method to ours (screening of $\sim 400$ mutations in 33 cancer genes), MacComaill et al. (17) identified at least one somatic mutation in 37\% out of 903 cancer tissues from 12 different sites. The genomic approach used in this study allowed us to recognize two previously identified CTNNB1 mutations, validating the sensitivity of the method. Interestingly, $R B 1$ emerged as a new candidate cancer gene, never investigated previously in ACCs.

The key aspects of the study are the discovery of $R B 1$ gene mutations in aggressive ACCs with the loss of the pRb. RB1 is a well-known gatekeeper tumor suppressor gene, whose loss or inactivation leads to uncontrolled cell proliferation (18). RB1 is mutated in various types of cancer with tissue specificity. In the COSMIC database, the highest RB1 mutation frequencies are found in eye (28.9\%), urinary tract (19.6\%), large intestine (17.3\%), and bone $(12.3 \%)$ carcinomas. RB1 mutations are less frequent in endometrium (8.7\%), lung (6\%), salivary gland $(5 \%)$, CNS $(4.2 \%)$, liver $(3.9 \%)$, ovary $(3.1 \%)$, and pancreas $(1.5 \%)$ carcinomas. The $R B 1$ mutation rate in ACCs remained unknown. With a $6 \%$ mutational frequency, or $10 \%$ if one considers only the ACC group with a poor prognosis, $R B 1$ should be viewed as an actor of ACC development. The loss of $\mathrm{pRb}$ expression was even more prevalent (27\%) in the aggressive ACCs. Somatic mutations and $\mathrm{LOH}$ are the two mechanisms of $R B 1$ inactivation identified in this study. Small deletions (missed by direct sequencing and $\mathrm{LOH}$ study)

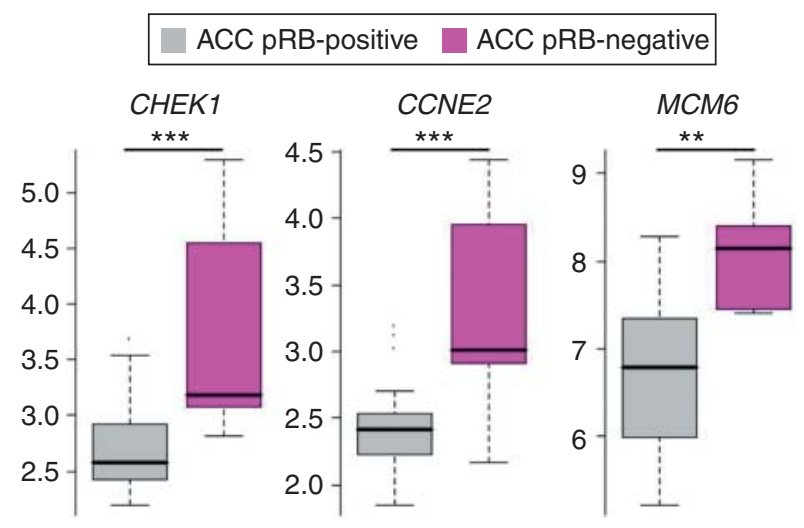

\section{Figure 5}

Expression of three representative $\mathrm{pRB}$-negative target genes in ACCs. Three examples of pRB targets (CHEK1, CCNE2, and MCM6). Each panel contains two box plots representing the distributions of the log intensity values (microarray data) for the ACC pRB-positive (gray, $n=24$ ) and ACC pRB-negative (pink, $n=5$ ). ${ }^{*} P<0.01$ and $* * * P<0.001$ (Limma test). 
encompassing one of the 27 exons of $R B 1$ may be another mechanism explaining the loss of $\mathrm{pRb}$. Translocations, alterations in regulatory regions or deep intronic mutations are other possibilities. It is worth noting that in $3-4 \%$ of retinoblastomas, only one $R B 1$ mutation could be identified, suggesting an independent mechanism (19).

$R B 1$ is another gene after TP53 and CTNNB1 for which recurrent abnormalities are identified in ACCs. It is worth mentioning that in the human ACC-derived NCI-H295R cell line, somatic mutations in only three genes have been identified. TP53 and CTNNB1 are already known to play a crucial role in adrenal carcinogenesis, and the third mutation involves RB1, inactivated through homozygous deletion (COSMIC mutation ID: 19554, c.862_2787del1926). It provides further evidence in support of a role for $R B 1$ in adrenocortical malignancy.

As $R B 1$ abnormalities were only found in the subgroup of aggressive ACCs, it might suggest that $R B 1$ loss could be a late event in ACC. This has been demonstrated in resistant prostate cancer (13). Another possibility could be the necessity to inactivate in the same tumor the p53 and $\mathrm{pRb}$ pathways. In that regard, it should be noted that four ACCs had a simultaneous alteration of both genes. Cooperation between these two pathways has been demonstrated in different human cancers $(20,21)$.

To conclude, this study demonstrates for the first time $\mathrm{pRb}$ loss of expression in ACCs, and specifically in the subgroup of poor-prognosis ACCs. These findings point to a role of the $\mathrm{pRb}$ pathway in ACCs. The overall prevalence of mutations in ACC suggests the involvement of yet unidentified oncogenes or other molecular mechanisms of adrenal carcinogenesis. As somatic alterations affecting the $\mathrm{pRb}$ pathway generally occur with a mutually exclusive pattern $(20,22)$, it can be anticipated that with the use of the next-generation sequencing, other abnormalities of this pathway will be reported. The combination of genomic approaches, including exome sequencing, SNP array genotyping, DNA methylation analyses, mRNA expression array data, and miRNA sequencing will allow to have an integrative and extensive molecular characterization of the disease, as reported in other cancers $(23,24,25)$. In this respect, comparative genomic hybridization reported amplification of the $C D K 4$ oncogene and deletion of the $C D K N 2 A$ and $C D K N 2 B$ tumor suppressor genes, which are known actors of the $\mathrm{Rb}$ pathway (26).

Supplementary data

This is linked to the online version of the paper at http://dx.doi.org/10.1530/ EJE-13-0778.
Declaration of interest

The authors declare that there is no conflict of interest that could be perceived as prejudicing the impartiality of the research reported.

\section{Funding}

This study was supported in part by the COMETE Network (Programme Hospitalier de Recherche Clinique Grant AOM95201), the INCa Recherche Translationelle 2009-RT-02, the Institut National de la Santé et de la Recherche Médicale (G Assié is receiving a Contrat d'Interface), and the Conny-Maeva Charitable Foundation.

\section{Acknowledgements}

The authors thank the sequencing platform of Cochin Institute and the tumor bank of Cochin Hospital (Prof. B Terris) for their help in collection of samples, as well as all the members of the Genomics and Signaling of Endocrine Tumors team.

\section{References}

1 Lughezzani G, Sun M, Perrotte P, Jeldres C, Alasker A, Isbarn H, Budaus L, Shariat SF, Guazzoni G, Montorsi F et al. The European Network for the Study of Adrenal Tumors staging system is prognostically superior to the international union against cancer-staging system: a North American validation. European Journal of Cancer 2010 46 713-719. (doi:10.1016/j.ejca.2009.12.007)

2 de Reynies A, Assie G, Rickman DS, Tissier F, Groussin L, Rene-Corail F, Dousset B, Bertagna X, Clauser E \& Bertherat J. Gene expression profiling reveals a new classification of adrenocortical tumors and identifies molecular predictors of malignancy and survival. Journal of Clinical Oncology 200927 1108-1115. (doi:10.1200/JCO.2008.18.5678)

3 Giordano TJ, Kuick R, Else T, Gauger PG, Vinco M, Bauersfeld J, Sanders D, Thomas DG, Doherty G \& Hammer G. Molecular classification and prognostication of adrenocortical tumors by transcriptome profiling. Clinical Cancer Research 200915 668-676. (doi:10.1158/1078-0432.CCR-08-1067)

4 Gicquel C, Bertagna X, Gaston V, Coste J, Louvel A, Baudin E, Bertherat J, Chapuis Y, Duclos JM, Schlumberger M et al. Molecular markers and long-term recurrences in a large cohort of patients with sporadic adrenocortical tumors. Cancer Research 200161 6762-6767.

5 Libe R, Groussin L, Tissier F, Elie C, Rene-Corail F, Fratticci A, Jullian E, Beck-Peccoz P, Bertagna X, Gicquel C et al. Somatic TP53 mutations are relatively rare among adrenocortical cancers with the frequent $17 \mathrm{p} 13$ loss of heterozygosity. Clinical Cancer Research 200713 844-850. (doi:10.1158/1078-0432.CCR-06-2085)

6 Tissier F, Cavard C, Groussin L, Perlemoine K, Fumey G, Hagnere AM, Rene-Corail F, Jullian E, Gicquel C, Bertagna X et al. Mutations of $\beta$-catenin in adrenocortical tumors: activation of the Wnt signaling pathway is a frequent event in both benign and malignant adrenocortical tumors. Cancer Research 200565 7622-7627. (doi:10.1158/ 0008-5472.CAN-05-0593)

7 Gaujoux S, Grabar S, Fassnacht M, Ragazzon B, Launay P, Libe R, Chokri I, Audebourg A, Royer B, Sbiera S et al. $\beta$-Catenin activation is associated with specific clinical and pathologic characteristics and a poor outcome in adrenocortical carcinoma. Clinical Cancer Research 201117 328-336. (doi:10.1158/1078-0432.CCR-10-2006)

8 Ragazzon B, Libe R, Gaujoux S, Assie G, Fratticci A, Launay P, Clauser E, Bertagna X, Tissier F, de Reynies A et al. Transcriptome analysis reveals that p53 and $\{\beta\}$-catenin alterations occur in a group of aggressive adrenocortical cancers. Cancer Research 201070 8276-8281. (doi:10. 1158/0008-5472.CAN-10-2014) 
9 Jurinke C, Oeth P \& van den Boom D. MALDI-TOF mass spectrometry: a versatile tool for high-performance DNA analysis. Molecular Biotechnology 200426 147-164. (doi:10.1385/MB:26:2:147)

10 Thomas RK, Baker AC, Debiasi RM, Winckler W, Laframboise T, Lin WM, Wang M, Feng W, Zander T, MacConaill L et al. Highthroughput oncogene mutation profiling in human cancer. Nature Genetics 200739 347-351. (doi:10.1038/ng1975)

11 Vivante A, Amariglio N, Koren-Michowitz M, Ashur-Fabian O, Nagler A, Rechavi G \& Cohen Y. High-throughput, sensitive and quantitative assay for the detection of BCR-ABL kinase domain mutations. Leukemia 200721 1318-1321. (doi:10.1038/sj.leu.2404635)

12 Houdayer C, Gauthier-Villars M, Lauge A, Pages-Berhouet S, Dehainault C, Caux-Moncoutier V, Karczynski P, Tosi M, Doz F, Desjardins $\mathrm{L}$ et al. Comprehensive screening for constitutional RB1 mutations by DHPLC and QMPSF. Human Mutation 200423 193-202. (doi:10.1002/humu.10303)

13 Sharma A, Yeow WS, Ertel A, Coleman I, Clegg N, Thangavel C, Morrissey C, Zhang X, Comstock CE, Witkiewicz AK et al. The retinoblastoma tumor suppressor controls androgen signaling and human prostate cancer progression. Journal of Clinical Investigation 2010 120 4478-4492. (doi:10.1172/JCI44239)

14 Houdayer C, Dehainault C, Mattler C, Michaux D, Caux-Moncoutier V, Pages-Berhouet S, d'Enghien CD, Lauge A, Castera L, Gauthier-Villars M et al. Evaluation of in silico splice tools for decision-making in molecular diagnosis. Human Mutation 2008 29 975-982. (doi:10.1002/humu.20765)

15 Houdayer C, Caux-Moncoutier V, Krieger S, Barrois M, Bonnet F, Bourdon V, Bronner M, Buisson M, Coulet F, Gaildrat P et al. Guidelines for splicing analysis in molecular diagnosis derived from a set of 327 combined in silico/in vitro studies on BRCA1 and BRCA2 variants. Human Mutation 201233 1228-1238. (doi:10.1002/humu.22101)

16 Abidi O, Knari S, Sefri H, Charif M, Senechal A, Hamel C, Rouba H, Zaghloul K, El Kettani A, Lenaers G et al. Mutational analysis of the RB1 gene in Moroccan patients with retinoblastoma. Molecular Vision 2011 17 3541-3547.

17 MacConaill LE, Campbell CD, Kehoe SM, Bass AJ, Hatton C, Niu L, Davis M, Yao K, Hanna M, Mondal C et al. Profiling critical cancer gene mutations in clinical tumor samples. PLoS ONE 20094 e7887. (doi:10. 1371/journal.pone.0007887)

18 Bremner R \& Zacksenhaus E. Cyclins, Cdks, E2f, Skp2, and more at the first International RB Tumor Suppressor Meeting. Cancer Research 2010 70 6114-6118. (doi:10.1158/0008-5472.CAN-10-0358)

19 Rushlow DE, Mol BM, Kennett JY, Yee S, Pajovic S, Theriault BL, Prigoda-Lee NL, Spencer C, Dimaras H, Corson TW et al. Characterisation of retinoblastomas without RB1 mutations: genomic, gene expression, and clinical studies. Lancet Oncology 201314 327-334. (doi:10.1016/S1470-2045(13)70045-7)

20 Sherr CJ \& McCormick F. The RB, and p53 pathways in cancer. Cancer Cell 20022 103-112. (doi:10.1016/S1535-6108(02)00102-2)

21 Conkrite K, Sundby M, Mu D, Mukai S \& MacPherson D. Cooperation between $\mathrm{Rb}$ and Arf in suppressing mouse retinoblastoma. Journal of Clinical Investigation 2012122 1726-1733. (doi:10.1172/JCI61403)

22 Macleod KF. The RB, tumor suppressor: a gatekeeper to hormone independence in prostate cancer? Journal of Clinical Investigation 2010 120 4179-4182. (doi:10.1172/JCI45406)

23 Richter J, Schlesner M, Hoffmann S, Kreuz M, Leich E, Burkhardt B, Rosolowski M, Ammerpohl O, Wagener R, Bernhart SH et al. Recurrent mutation of the ID3 gene in Burkitt lymphoma identified by integrated genome, exome and transcriptome sequencing. Nature Genetics 201244 1316-1320. (doi:10.1038/ng.2469)

24 Kandoth C, Schultz N, Cherniack AD, Akbani R, Liu Y, Shen H, Robertson AG, Pashtan I, Shen R, Benz CC et al. Integrated genomic characterization of endometrial carcinoma. Nature 2013497 67-73. (doi:10.1038/nature12113)

25 Sato Y, Yoshizato T, Shiraishi Y, Maekawa S, Okuno Y, Kamura T, Shimamura T, Sato-Otsubo A, Nagae G, Suzuki H et al. Integrated molecular analysis of clear-cell renal cell carcinoma. Nature Genetics 201345 860-867. (doi:10.1038/ng.2699)

26 De Martino MC, Al Ghuzlan A, Aubert S, Assie G, Scoazec JY, Leboulleux S, Do Cao C, Libe R, Nozieres C, Lombes M et al. Molecular screening for a personalized treatment approach in advanced adrenocortical cancer. Journal of Clinical Endocrinology and Metabolism 201398 4080-4088. (doi:10.1210/jc.2013-2165)

Received 24 September 2013

Revised version received 3 December 2013

Accepted 17 December 2013 